\title{
Der frühe Beginn der Zwangsstörung: Einfluss auf Symptomatik und Schweregrad
}

\author{
Petra Jänscha \\ Willi Butollob \\ Michael Zaudiga \\ Gisela Röper ${ }^{b}$ Walter Hauke ${ }^{a}$ \\ Christoph Piesbergen ${ }^{b}$ \\ a Psychosomatische Klinik Windach am Ammersee, \\ ${ }^{b}$ Department Psychologie, Klinische Psychologie und Psychotherapie, Ludwig-Maximilians-Universität, München, Deutschland
}

\section{Schlüsselwörter \\ Zwangsstörung · Yale-Brown Obsessive Compulsive Scale $\cdot$ Störungsbeginn}

\section{Zusammenfassung}

Einleitung: Die vorliegende Untersuchung geht der Fragestellung nach, ob sich eine Zwangsstörung, die bereits im Kindes- bzw. Jugendalter beginnt, von einer Zwangsstörung, die erst im Erwachsenenalter beginnt, hinsichtlich Schweregrad und Symptomatik unterscheidet. Patienten und Methoden: Eine Stichprobe von 370 Patienten mit Zwangsstörung (ICD-10 F42), die sich zwischen 1998 und 2002 stationär in der Psychosomatischen Klinik Windach befanden, wurde in eine Early-Onset-Gruppe (Störungsbeginn $\leq 15$ Jahre) und in eine Late-OnsetGruppe (Störungsbeginn $\geq 16$ Jahre) aufgeteilt. Die Gruppen wurden über ICD-10-Diagnosen und Y-BOCSWerte verglichen. Ergebnisse: Beim Schweregrad zeigte sich, dass 20,5\% der Early-Onset-Gruppe, aber lediglich $8,7 \%$ der Late-Onset-Gruppe unter einer "massiven Zwangsstörung" leiden. Bei der Symptomatik zeigte sich, dass die Early-Onset-Gruppe häufiger die Diagnose "Zwangsgedanken und -handlungen gemischt" (76,9\%) erhält als die Late-Onset-Gruppe (61,8\%). Außerdem nennt die Early-Onset-Gruppe sowohl für die Gegenwart als auch für die Vergangenheit mehr Symptome als die Late-Onset-Gruppe (Gegenwart 8,2 vs. 7,0; Vergangenheit 5,5 vs. 3,9 Symptomgruppen). Weiter ergaben sich inhaltliche Unterschiede der Zwangsgedanken und Zwangshandlungen. Schlussfolgerungen: Early-OnsetPatienten scheinen häufiger von einer massiven Form der Zwangsstörung und einer größeren Symptomvielfalt betroffen zu sein als Late-Onset-Patienten. Ob es sich bei der Zwangsstörung mit Beginn im Kindes- und Jugendalter um einen abgrenzbaren Subtypus handelt, konnte jedoch in dieser Untersuchung nicht eindeutig geklärt werden und bedarf weiterer Forschungen.

\author{
Key Words \\ Obsessive Compulsive Disorder · Yale-Brown Obsessive \\ Compulsive Scale · Disorder onset
}

\section{Summary}

The Early Onset of Obsessive-Compulsive Disorder and Its Impact on Symptomatology and Severity

Introduction: This study investigates if obsessive compulsive disorder with early onset differs in severity and symptomatology from that with late onset. Patients and Methods: A sample of 370 patients with obsessive compulsive disorder (OCD; ICD 10 F42) who received in-patient treatment at the psychosomatic clinic of Windach between 1998 and 2002 were divided into an early-onset group (onset $\leq 15$ years) and a late-onset group (onset $\geq 16$ years). Groups were compared regarding ICD-10 diagnosis and Y-BOCS scores. Results: Considering severity of the disorder $20.5 \%$ of the early-onset group but merely $8.7 \%$ of the late-onset group suffered from an extreme form of OCD. With respect to symptomatology, the early-onset group was diagnosed with 'obsessions and compulsions, mixed' (76.9\%) more often than the lateonset group (61.8\%). Also, the early-onset group reported a wider variety of symptoms both for the present and for the past than the late-onset group (present 8,2 vs 7.0; past 5.5 vs 3.9 types of symptoms). There were also differences in the content of rumination and types of compulsive rituals. Conclusions: Patients with early-onset OCD seem to be more frequently affected by an extreme form of OCD and to experience a higher variety of symptoms than patients with late-onset OCD. If early-onset OCD can be considered a distinct subtype could not be answered unequivocally by the results of this study. This question needs additional research.

\begin{tabular}{ll}
\hline KARGER & @ 2007 S. Karger GmbH, Freiburg \\
$\begin{array}{l}\text { Fax +49 76145207 14 } \\
\begin{array}{l}\text { E-mail Information@Karger.de } \\
\text { www.karger.com }\end{array}\end{array}$ & $\begin{array}{l}\text { Accessible online at: } \\
\text { www.karger.com/ver }\end{array}$
\end{tabular}




\section{Einleitung}

Therapiemöglichkeiten $\mathrm{zu}$ optimieren und therapeutische Misserfolge zu minimieren ist Sinn der Therapieforschung [Althaus et al., 2000; Aigner et al., 2004; Roth et al., 2004]. Seit einigen Jahren ist auch die Bestimmung von Subgruppen mit dem Ziel einer differentiellen Indikation bei frühem bzw. spätem Störungsbeginn in der Diskussion. Unterschiedliche Cutoff-Werte machen die Integration verschiedener Ergebnisse jedoch schwierig, daher müssen bisherige Ergebnisse als vorläufig betrachtet werden. Die Bestimmung einer sinnvollen Altersgrenze zwischen frühem bzw. spätem Störungsbeginn sollte daher mit weiteren Untersuchungen einhergehen. Das Durchschnittsalter für den Beginn einer Zwangserkrankung wird in den meisten Studien mit 22-23 Jahren angegeben [Reinecker, 1994; Reinecker und Zaudig, 1995], im angelsächsischen Raum mit dem Zeitraum zwischen später Adoleszenz und frühem Erwachsenenalter [Rachman und Hodgson, 1980]. Nach Karno et al. [1988] liegt die größte Anzahl von Erstmanifestationen bei 20,9-25,4 Jahren. Laut Lakatos und Reinecker [1999] beginnt jedoch bei einem Fünftel der Patienten die Störung in der Kindheit und beim Gros der Betroffenen in der Pubertät. Nach Rapoport [1986] geben ein Drittel bis die Hälfte der erwachsenen Patienten an, schon als Kind unter den Symptomen der Zwangsstörung gelitten zu haben. Geller et al. [1998] sprechen von einer bimodalen Altersverteilung bei Erstmanifestation der Erkrankung mit einem ersten Gipfel in der Kindheit und einem zweiten im jungen Erwachsenenalter. Daraus ergibt sich die Frage, ob die Zwangsstörung bei Kindern/Jugendlichen der gleichen nosologischen Entität entspricht wie die erwachsene Form der Zwangsstörung.

Bei einer weiteren Untersuchung verglichen Geller et al. [2001] eine Gruppe aus dem kinder- und jugendpsychiatrischen Bereich und eine aus dem erwachsenenpsychiatrischen Bereich. Sie fanden Ähnlichkeiten von Prävalenz und Phänomenologie der Zwangsstörung bei Jugendlichen und Erwachsenen. Als Cutoff-Wert für einen frühen Störungsbeginn hatten sie 12 Jahre festgelegt. Männliche Kinder und Jugendliche litten häufiger unter einer akuten Zwangsstörung als weibliche; ein Ergebnis, das auch die Studien von Fontenelle et al. [2003] und Jaisoorya et al. [2003] erbrachten, jedoch bei Cutoff-Werten von $>17$ bzw. $>18$ Jahren. Rosario-Campos et al. [2001] fanden hingegen keine unterschiedliche Geschlechterverteilung bei Kindern/Jugendlichen oder Erwachsenen. Geller et al. [2001] berichten weiter, dass die «Einsicht in die Unsinnigkeit der Rituale» bei Kindern deutlich geringer ausgeprägt war und sie weniger unter Zwangsgedanken mit sexuellem Inhalt litten als Jugendliche und Erwachsene, aber mehr Rituale zeigten, die sich auf Sammeln und Horten ausrichteten. Kinder und Jugendliche nannten häufiger als Erwachsene Zwangsgedanken mit aggressiven Inhalten. Übereinstimmung bestand im Ansprechen auf medikamentöse Behand- lung mit serotonerg wirkenden Substanzen. Die Autoren schließen, dass es sich bei der Zwangsstörung mit Erstmanifestation in der Kindheit um einen Entwicklungssubtyp der Zwangsstörung handelt, was in der Forschung, der Behandlung der Störung und im öffentlichen Gesundheitswesen berücksichtigt werden solle.

Rosario-Campos et al. [2001] fanden bei Early-Onset-Patienten $(<10)$ eine schwerere Ausprägung der Störung, die auf einen signifikant höheren Y-BOCS-Wert in der Subskala Zwangshandlung zurückzuführen war. Auf Symptomebene zeigte sich, dass Early-Onset-Patienten häufiger unter «Ticähnlichen» Zwangshandlungen litten als die Vergleichsgruppe. Dies ergab auch die Untersuchung von Millet et al. [2004], die bei Early-Onset-Patienten $(<15)$ häufiger spezifische Zwangsinhalte wie Aberglaube, magische Gedanken, Verschmutzung sowie Sammel- und Zählzwänge fanden.

Zwei weitere Forschergruppen legten als Cutoff für einen frühen vs. späten Störungsbeginn das 18. Lebensjahr fest. Bei Sobin et al. [2000] zeigten Erwachsene mit frühem Störungsbeginn häufiger Gedankenzwänge in Bezug auf Symmetrie, Religion, Aberglaube und Befürchtungen bezüglich des eigenen Körpers sowie häufiger Handlungszwänge in Form von Kontroll- und Zählzwängen. Darüber hinaus war die Anzahl bestehender Symptome bei Patienten mit frühem Störungsbeginn höher als bei Patienten mit spätem Störungsbeginn, und sie litten häufiger unter Wasch- und Berührungszwängen. Jaisoorya et al. [2003] fanden bei Jugendlichen und Erwachsenen mit frühem Störungsbeginn häufiger Zwangsgedanken über Kontaminationsgefahren und Kontrollzwänge.

In der Windacher Follow-up-Studie [Reinecker und Zaudig, 1995] hing der Schweregrad der Zwangsstörung weder vom Alter bei Störungsbeginn noch von der Störungsdauer ab. Der klinischen Erfahrung nach war der Schweregrad der Zwangsstörung jedoch umso größer, je jünger die Patienten bei Erstmanifestation der Zwänge waren.

Die vorliegende Studie soll zur Klärung folgender Fragen beitragen: Wenn es einen Entwicklungssubtyp der Zwangsstörung bei Kindern/Jugendlichen gibt, geht dieser bei Erwachsenen in eine «normale» Zwangsstörung über, oder unterscheiden sich Zwangsstörungen bei Erwachsenen je nachdem, ob sie früh oder spät begonnen haben? Im Einzelnen werden folgende Fragestellungen untersucht: (1) Bestätigt sich die in der Literatur beschriebene bimodale Kurve mit einem Häufigkeitsgipfel in der Kindheit und einem im frühen Erwachsenenalter? (2) Bestätigt sich der in der Literatur beschriebene höhere männliche Anteil unter den Patienten mit frühem Störungsbeginn? (3) Unterscheiden sich Patienten, deren Zwangsstörung im Kindes-/Jugendalter begonnen hat, von Patienten, deren Störungen im Erwachsenenalter begonnen hat hinsichtlich des Schweregrads der Erkrankung? (4) Unterscheiden sich Patienten, deren Zwangsstörung im Kindes-/ Jugendalter begonnen hat, von den Patienten, deren Störungen im Erwachsenenalter begonnen hat hinsichtlich der Symptomatik der Erkrankung? 


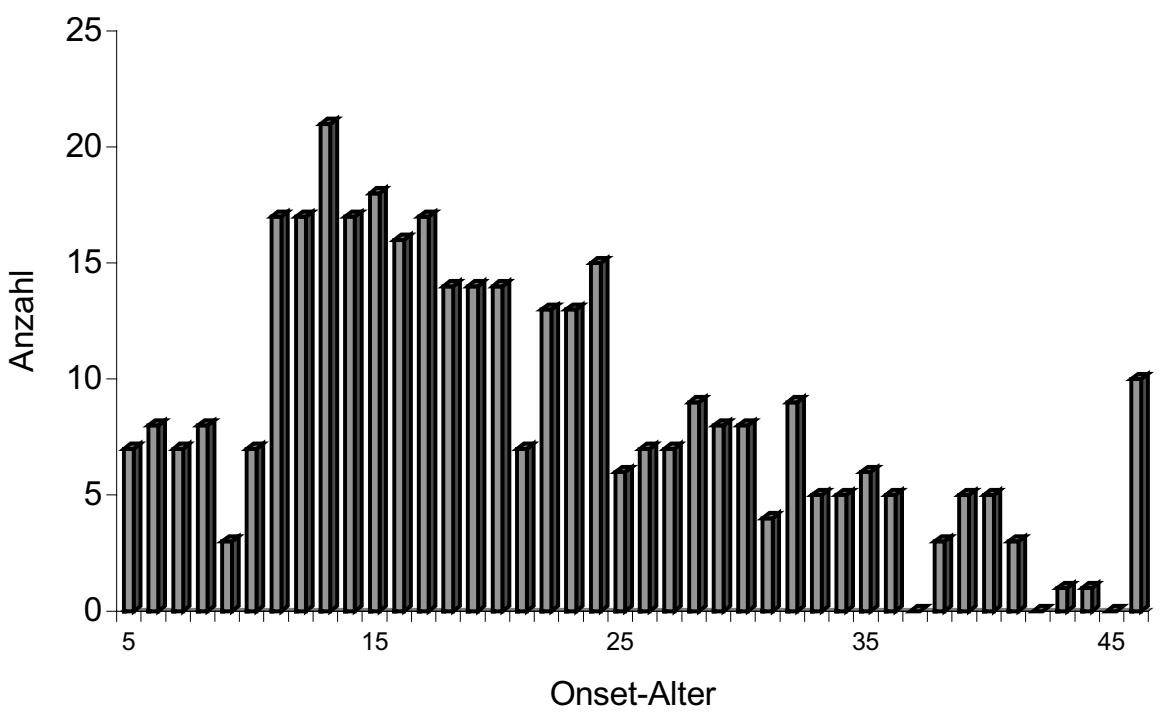

\section{Patienten und Methoden}

In der vorliegenden Untersuchung wurde als Cutoff für einen frühen oder späten Störungsbeginn a priori 15 Jahre festgelegt. Dementsprechend bilden die Patienten, deren Zwangsstörung mit maximal 15 Jahren begann, die Early-Onset-Gruppe und Patienten, deren Störung mit 16 Jahren oder später begann, die Late-Onset-Gruppe. Definiert wird der Beginn der Zwangsstörung durch das erstmalige Auftreten von Zwangssymptomen mit den typischen begleitenden Angst- und Spannungsgefühlen (nach der Erinnerung des Betreffenden) [vgl. Jaisoorya et al., 2003; Rosario-Campos et al., 2001].

Bei der Festlegung der Altersgrenze ließen wir uns von entwicklungspsychologischen Phasenmodellen leiten [Erikson, 1966; Kegan, 1986; Noam, 1997]. Vor dem 15. Lebensjahr dürfte sich kaum ein Jugendlicher in einer Entwicklungsphase befinden, die dem jungen (post-konformistischen) Erwachsenenalter zuzuordnen wäre [Loevinger, 1976]. Der frühe Störungsbeginn wird hier also in die Phase der Kindheit einschließlich Adoleszenz gelegt.

Die Symptomatik der Zwangsstörung wird anhand der ICD-10-Diagnosen aus der Basisdokumentation (F42.0 «vorwiegend Zwangsgedanken»; F42.1 «vorwiegend Zwangshandlungen» und F42.2 «Zwangsgedanken und Zwangshandlungen, gemischt») abgebildet. Weiter kommt die YBOCS Symptom-Checkliste [Goodman et al., 1989] zum Einsatz, die 70 Items zu Zwangsgedanken und Zwangshandlungen enthält, nach deren gegenwärtigem bzw. früherem Vorhandensein gefragt wird. Diese 70 Items sind 15 Hauptgruppen zugeordnet, die bezüglich der Häufigkeit der Nennungen zwischen der Early-Onset-Gruppe und der Late-Onset-Gruppe zum gegenwärtigen Zeitpunkt bzw. zum früheren Zeitpunkt miteinander verglichen werden. Darüber hinaus wird verglichen, wie viele Symptomgruppen die Early-Onset-Gruppe und die Late-Onset-Gruppe durchschnittlich nennen. Der Schweregrad der Zwangsstörung wird zum einen durch den Gesamtscore der Y-BOCS bei Aufnahme in die Klinik abgebildet, zum anderen werden die Werte in den Y-BOCS-Untertests «Schweregrad der Zwangsgedanken» und «Schweregrad der Zwangshandlungen» verglichen.

Entsprechend dem jeweiligen Skalenniveau werden zweiseitige t-Tests oder $\mathrm{Chi}^{2}$-Tests durchgeführt. Als Signifikanzniveau wurde $\mathrm{p}=0,05$ festgelegt. Bei signifikanten Ergebnissen in den t-Tests wurden Effektstärken nach Cohen [1988] berechnet. Auf eine Alpha-Fehler-Korrektur nach Bonferroni oder Holm [1979] wurde verzichtet, da die Studie eher explorativ und Hypothesen generierend ausgerichtet ist. Eine derartige Korrektur hätte die Wahrscheinlichkeit eines Beta-Fehlers stark erhöht. Das kann nicht im Sine einer Untersuchung sein, deren Ziel es ist, mögliche Effekte nicht zu übersehen, über deren Realität die weitere Forschung zu entscheiden hat. Somit soll der Begriff «Signifikanz» nicht im statistischen
Sinne einer verallgemeinernden, induktiven Aussage gesehen werden, sondern mehr als Kriterium für die Aussagekraft oder Bedeutsamkeit eines Testergebnisses in unserer Stichprobe.

\section{Stichprobe}

Die Untersuchung basiert auf einer Stichprobe von 370 Patienten, die zwischen Januar 1998 und Dezember 2002 stationär in der Psychosomatischen Klinik Windach aufgrund der Erstdiagnose einer Zwangsstörung (F42) aufgenommen wurden. Die diagnostische Einordnung erfolgte durch erfahrene Kliniker nach den Kriterien des ICD-10. Patienten, die im genannten Zeitraum mehrmals stationär aufgenommen wurden, gehen nur einmal (erste Aufnahme) in die Untersuchung ein.

Von 339 Patienten lagen außer der ICD-Diagnostik auch Datensätze der Y-BOCS und von 192 Patienten auch Datensätze der Y-BOCS SymptomCheckliste vor. Dem Fehlen oder Vorhandensein der Y-BOCS SymptomCheckliste liegt keine Systematik zugrunde.

Die Gesamtstichprobe besteht aus 201 Frauen $(54,3 \%)$ und 169 Männern (45,7\%). Das durchschnittliche Alter bei Aufnahme liegt bei $\mathrm{M}=34,8 \pm$ 11,35 Jahren (Range 17-75). Das Alter bei Erstmanifestation der Zwangsstörung liegt bei $\mathrm{M}=21,1 \pm 10,63$ Jahren (Range 5-65). Der Median liegt bei 19, der Modalwert bei 13 Jahren. Männer erkranken im Mittel mit $19,7 \pm 9,44$ Jahren, Frauen etwas später mit 22,3 $\pm 11,28$. Bei 130 Patienten $(35,1 \%)$ begann die Störung eigenen Angaben zufolge mit 15 oder weniger Jahren, bei 233 Patienten $(63,0 \%)$ mit 16 oder mehr Jahren. Bei 7 Patienten $(1,9 \%)$ ist das Alter bei Störungsbeginn nicht zweifelsfrei zu bestimmen. Ohne Berücksichtigung dieser Patienten liegt die Verteilung von Early-Onset zu Late-Onset bei 35,8 zu $64 \%$.

101 Patienten $(28,4 \%)$ haben einen Haupt- bzw. Volksschulabschluss, 115 Patienten (32,3\%) die Mittlere Reife, den Realschulabschluss oder die Polytechnische Hochschule. Abitur bzw. Fachabitur haben 118 Patienten $(33,1 \%) .8$ Patienten $(2,2 \%)$ gehen noch zur Schule, 9 Patienten $(2,5 \%)$ haben keinen Schulabschluss, $2(0,6 \%)$ den Sonderschulabschluss und 3 Patienten $(0,8 \%)$ geben einen «sonstigen» Schulabschluss an. Bei $14 \mathrm{~Pa}$ tienten finden sich keine Angaben zum Schulabschluss. Von der Gesamtstichprobe geben 11 Patienten $(3,1 \%)$ an, bereits in der Kindheit einen Kliniker wegen der Zwangssymptome konsultiert zu haben.

\section{Instrumente}

Aus den Krankenakten der oben beschriebenen Stichprobe werden folgende Instrumente bei dieser Untersuchung verwendet:

- Basisdokumentation: genaue Diagnosen nach ICD-10 und demographische Angaben wie Familienstand, Schulbildung, Beruf, Erkrankungsdauer, frühere und jetzige Behandlungen, Therapiedauer, Medikation bei Aufnahme und Entlassung. 
- Y-BOCS [Goodman et al., 1989; deutsch: Hand und Büttner-Westphal, 1991]. Ratingverfahren zur Quantifizierung des Schweregrades der Zwangsstörung.

- Y-BOCS Symptom-Checkliste [Goodman et al., 1989]. Ratingverfahren zur qualitativen Spezifizierung der Zwangsstörung.

\section{Ergebnisse}

Alter bei Störungsbeginn

In der Early-Onset-Gruppe $(\mathrm{n}=130)$ begann die Störung durchschnittlich mit 11,3 \pm 3,0 Jahren (Median 12,0), in der Late-Onset-Gruppe $(\mathrm{n}=233)$ mit 26,5 \pm 9,3 Jahren (Median 24,0). Abbildung 1 zeigt nahezu eine Gleichverteilung des Störungsbeginns bis zum Alter von 10 Jahren (mit einem Einbruch im Alter von 9 Jahren). Ab dem 11. Lebensjahr kommt es zu einem sprunghaften Anstieg des Störungsbeginns mit einem Häufigkeitsgipfel bei 13 Jahren. Die Häufigkeit der Neumanifestationen bleibt auf diesem Niveau bis zu einem starken Einbruch nach dem 24. Lebensjahr. Die Zahl derer, die ihren Störungsbeginn nach dem 24. Lebensjahr datierten, wird sukzessive geringer. Die Verteilung des Alters bei Störungsbeginn in unserer Stichprobe weist im Gegensatz zu Geller et al. [1998] nicht auf eine zweigipflige Verteilung hin.

\section{Geschlechterverteilung}

In der Early-Onset-Gruppe liegt der Anteil der Frauen mit $48,5 \%(n=63)$ etwas niedriger als jener der Männer mit $51,5 \%(n=67)$. In der Late-Onset-Gruppe ist der Anteil der Frauen mit 57,5\% $(n=134)$ höher als jener der Männer mit 42,5\% ( $\mathrm{n}=99)$. Die Geschlechterverteilung zwischen den Gruppen mit frühem und spätem Störungsbeginn unterscheidet sich jedoch nicht signifikant. Angesichts der hohen Zahl an Probanden kann man davon ausgehen, dass das Ergebnis nicht auf eine zu niedrige Teststärke zurückzuführen ist.

\section{Schweregrad der Zwangsstörung}

Die mittleren Y-BOCS-Werte der beiden Gruppen divergieren nur leicht (Early-Onset-Gruppe: $24,7 \pm 7,35$; Late-OnsetGruppe: 23,50 $\pm 6,53$; n.s.). Beide Gruppen liegen im Mittel im Grenzbereich der «mäßigen zur schweren» Zwangsstörung. Im Y-BOCS-Untertest «Schweregrad der Zwangsgedanken» (möglicher Score: 0-20 Punkte) erreicht die Early-OnsetGruppe 13,33 \pm 3,97, die Late-Onset-Gruppe 12,20 \pm 4,22 Punkte (n.s.). Auch im Untertest «Schweregrad der Zwangshandlungen» (möglicher Score 0-20 Punkte) ergeben sich keine signifikanten Gruppenunterschiede (Early-Onset-Gruppe: 11,34 \pm 4,89; Late-Onset-Gruppe 11,93 $\pm 4,03)$.

Abbildung 2 zeigt, dass bei subklinischen (Score 0-7), leichten (Score 8-15) und mäßig ausgeprägten (Score 16-23) Zwängen die Early-Onset- und die Late-Onset-Gruppe annähernd gleich verteilt sind. Im Bereich der «schweren» Zwangsstörung (Score 24-31) finden sich 48\% $(\mathrm{n}=105)$ aus der LateOnset-Gruppe und 38\% ( $\mathrm{n}=44)$ aus der Early-Onset-Gruppe. Im Bereich der «extremen» Zwangsstörung (Score 32-40),

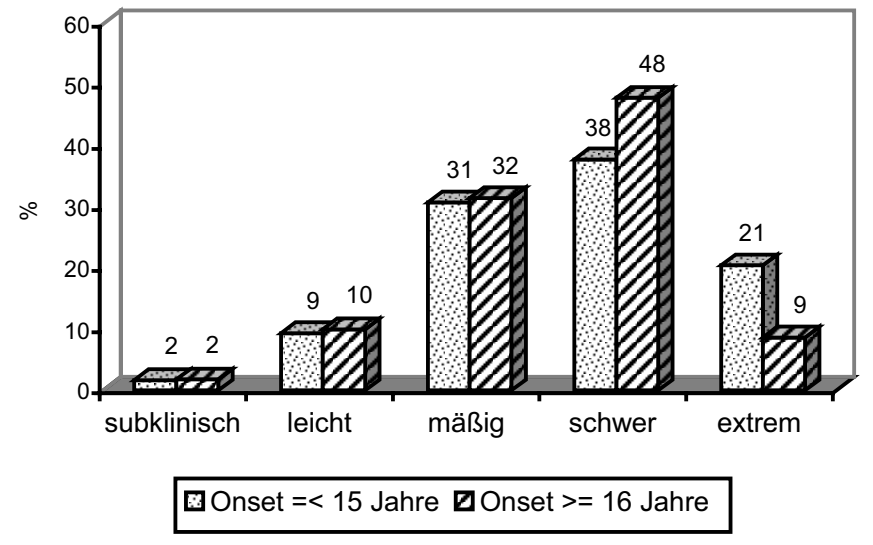

Abb. 2. Verteilung der Y-BOCS-Schweregradbereiche zwischen der Early-Onset- und der Late-Onset-Gruppe bei Aufnahme in die Klinik $(\mathrm{n}=336)$.

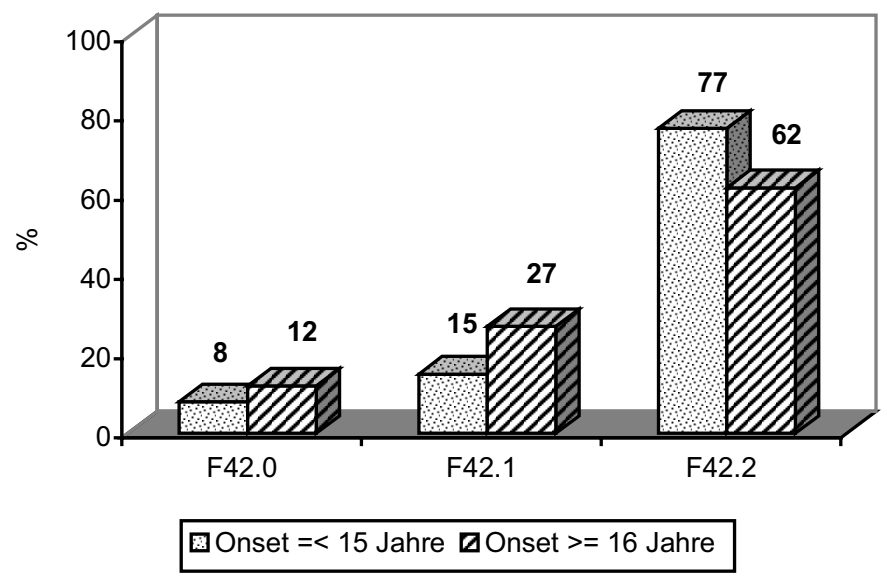

Abb. 3. Verteilung der Erstdiagnosen nach ICD-10 in der Early-Onsetund der Late-Onset-Gruppe (15-Jahre-Kriterium), n = 130 (F42.0 vorwiegend Zwangsgedanken; F42.1 vorwiegend Zwangshandlungen; F42.2 Zwangsgedanken und -handlungen, gemischt).

unter der 12,7\% der Gesamtstichprobe leiden, dreht sich das Verhältnis um: Hier finden sich $21 \%(\mathrm{n}=24)$ aus der Gruppe mit frühem Störungsbeginn, aber nur 9\% ( $=19)$ aus der Gruppe mit spätem Störungsbeginn. Dieser Unterschied ist signifikant $\left(\chi^{2}(1, \mathrm{~N}=336)=9,575 ; \mathrm{p}<0,05\right)$.

Somit kann man nicht schließen, dass eine Zwangsstörung mit frühem Beginn im Erwachsenenalter schwerer ausgeprägt ist als eine mit späterem Beginn. Allerdings scheinen - und das deckt sich mit dem klinischen Eindruck - Patienten mit frühem Störungsbeginn signifikant häufiger an einer «extremen» Zwangsstörung zu leiden als Patienten mit spätem Störungsbeginn.

\section{Symptomatik}

In der Early-Onset-Gruppe leiden 11 Patienten (8,5\%) unter «vorwiegend Zwangsgedanken» (ICD-10 F42.0), 19 (14,6\%) 
Tab. 1. Angaben in der Y-BOCS Symptom-Checkliste zu gegenwärtig vorhandenen Symptomen (Mehrfachnennungen möglich, $\mathrm{n}=192$ )

\begin{tabular}{|c|c|c|c|c|}
\hline & \multicolumn{2}{|c|}{$\begin{array}{l}\text { Störungsbeginn } \\
\leq 15 \text { Jahre }(\mathrm{n}=72)\end{array}$} & \multicolumn{2}{|c|}{$\begin{array}{l}\text { Störungsbeginn } \\
\geq 16 \text { Jahre }(\mathrm{n}=120)\end{array}$} \\
\hline & $\mathrm{n}$ & $\%$ & $\mathrm{n}$ & $\%$ \\
\hline Aggressive Zwangsgedanken & 52 & 72,2 & 79 & 65,8 \\
\hline Zwangsgedanken, die sich auf Verschmutzung beziehen & 55 & 76,4 & 83 & 69,2 \\
\hline Zwangsgedanken mit sexuellem Inhalt & 12 & 16,7 & 17 & 14,2 \\
\hline $\begin{array}{l}\text { Zwangsgedanken, die sich auf das Sammeln und Aufbew } \\
\text { von Gegenständen beziehen }\end{array}$ & 14 & 19,4 & 25 & 20,8 \\
\hline $\begin{array}{l}\text { Zwangsgedanken mit religiösen oder solchen Inhalten, d } \\
\text { schlechtes Gewissen erzeugen* }\end{array}$ & 36 & 50,0 & 39 & 32,5 \\
\hline $\begin{array}{l}\text { Zwangsgedanken, die sich auf Symmetrie oder Genauigk } \\
\text { beziehen }\end{array}$ & 26 & 36,1 & 37 & 30,8 \\
\hline Verschiedene Zwangsgedanken* & 62 & 86,1 & 82 & 68,3 \\
\hline Zwangsgedanken in Bezug auf den eigenen Körper & 40 & 55,6 & 54 & 45,0 \\
\hline Reinigungs-Wasch-Zwänge & 49 & 68,1 & 78 & 65,0 \\
\hline Kontrollzwänge & 61 & 84,7 & 97 & 80,8 \\
\hline Wiederholungszwänge & 51 & 70,8 & 77 & 64,2 \\
\hline Zählzwänge & 30 & 41,7 & 34 & 28,3 \\
\hline Ordnungszwänge & 23 & 31,9 & 34 & 28,3 \\
\hline Sammel- und Aufbewahrungszwänge & 21 & 29,2 & 29 & 24,2 \\
\hline Verschiedene Zwangshandlungen* & 55 & 76,4 & 71 & 59,2 \\
\hline
\end{tabular}

$* \mathrm{p}<0,05$. unter «vorwiegend Zwangshandlungen» (F42.1) und 100 (76,9\%) unter «Zwangsgedanken und Zwangshandlungen, gemischt» (F42.2). In der Late-Onset-Gruppe leiden 27 Patienten $(11,6 \%)$ unter «vorwiegend Zwangsgedanken», $62(26,6 \%)$ unter «vorwiegend Zwangshandlungen» und 144 $(61,8 \%)$ unter «Zwangsgedanken und -handlungen gemischt». Die Häufigkeit der gestellten Unterdiagnosen der Zwangsstörung unterscheidet sich signifikant $\left(\chi^{2}(2, N=363)=8,997\right.$; $\mathrm{p}<0,05)$. Die Early-Onset-Gruppe leidet häufiger an «Zwangsgedanken und -handlungen, gemischt» als die LateOnset-Gruppe (Abb. 3).

Alle in der Y-BOCS Symptom-Checkliste abgefragten Symptombereiche außer «Sammeln und Aufbewahren von Gegenständen» werden von Patienten mit frühem Krankheitsbeginn prozentual häufiger angegeben als von Patienten mit spätem Krankheitsbeginn. Für drei Bereiche wird der Unterschied signifikant (Tab. 1): Zwangsgedanken mit religiösem Inhalt oder solchen Inhalten, die ein schlechtes Gewissen erzeugen $\left(\chi^{2}(1, N=192)=5,790 ; p<0,05\right)$, verschiedene Zwangsgedanken $\left(\chi^{2}(1, \mathrm{~N}=192)=7,585 ; \mathrm{p}<0,05\right)$ sowie verschiedene Zwangshandlungen $\left(\chi^{2}(1, N=192)=5,917 ; \mathrm{p}<0,05\right)$.

Die Early-Onset-Gruppe gibt gegenwärtig vorhandene Symptome aus 8,15 \pm 3,11 Symptomgruppen an, während die LateOnset-Gruppe durchschnittlich 6,97 \pm 2,94 Symptomgruppen nennt. Dieser Unterschied ist signifikant (t $(190)=2,648$; $\mathrm{p}<0,05)$. Die Effektstärke beträgt 0,39. Somit bestätigt sich der Eindruck, dass die Gruppe mit frühem Störungsbeginn unter einer größeren Symptomvielfalt leidet als die Vergleichsgruppe mit spätem Störungsbeginn.

Auch bei den für die Vergangenheit berichteten Symptomen, nennt die Early-Onset-Gruppe alle Kategorien häufiger als die Late-Onset-Gruppe. Für fünf Bereiche wird der Unterschied signifikant (Tab. 2): Zwangsgedanken mit religiösem Inhalt oder solchen Inhalten, die ein schlechtes Gewissen erzeugen $\left(\chi^{2}(1, \mathrm{~N}=192)=4,851 ; \mathrm{p}<0,05\right)$, Zwangsgedanken in Bezug auf den eigenen Körper $\left(\chi^{2}(1, N=192)=3,872\right.$; $\mathrm{p}<0,05)$, Zählzwänge $\left(\chi^{2}(1, \mathrm{~N}=192)=5,146 ; \mathrm{p}<0,05\right)$, Ordnungszwänge $\left(\chi^{2}(1, \mathrm{~N}=192)=5,760 ; \mathrm{p}<0,05\right)$ sowie verschiedene Zwangshandlungen $\left(\chi^{2}(1, \mathrm{~N}=192)=9,484\right.$; $\mathrm{p}<0,05)$.

Die Early-Onset-Gruppe berichtet früher vorhandene Symptome aus durchschnittlich 5,48 \pm 4,43 Symptomgruppen, die Late-Onset-Gruppe Symptome aus 3,91 \pm 3,47 Symptomgruppen. Auch dieser Unterschied ist signifikant (t $(121)=2,559$; $\mathrm{p}<0,05)$. Die Effektstärke beträgt 0,40.

\section{Diskussion}

Die vorliegende Untersuchung soll zur Klärung beitragen, ob Unterschiede zwischen Zwangsstörungen bei Kindern/Jugendlichen und Zwangsstörungen bei Erwachsenen [Geller et al., 1998, 2001; Jaisoorya et al., 2003] entwicklungsbedingt sind, oder ob sie darauf hinweisen, dass es einen Subtyp der Zwangsstörung gibt, der in der Kindheit oder frühen Jugend beginnt und dessen phänotypische Unterschiede bis ins Erwachsenenalter hinein persistieren. Weder das Alter bei Störungsbeginn noch die Geschlechterverteilung der beiden Subgruppen können eine direkte Antwort auf diese Frage liefern. Vertiefte Kenntnisse über den Entwicklungspfad einer Stö- 
Tab. 2. Angaben in der Y-BOCS Symptom-Checkliste zu früher vorhandenen Symptomen (Mehrfachnennungen möglich, $\mathrm{n}=192$ )

\begin{tabular}{|c|c|c|c|c|}
\hline & \multicolumn{2}{|c|}{$\begin{array}{l}\text { Störungsbeginn } \\
\leq 15 \text { Jahre }(\mathrm{n}=72)\end{array}$} & \multicolumn{2}{|c|}{$\begin{array}{l}\text { Störungsbeginn } \\
\geq 16 \text { Jahre }(\mathrm{n}=120)\end{array}$} \\
\hline & $\mathrm{n}$ & $\%$ & $\mathrm{n}$ & $\%$ \\
\hline Aggressive Zwangsgedanken & 37 & 51,4 & 54 & 45,0 \\
\hline Zwangsgedanken, die sich auf Verschmutzung beziehen & 33 & 45,8 & 47 & 39,2 \\
\hline Zwangsgedanken mit sexuellem Inhalt & 11 & 15,3 & 10 & 8,3 \\
\hline \multicolumn{5}{|c|}{ Zwangsgedanken, die sich auf das Sammeln und Aufbewahren von } \\
\hline Gegenständen beziehen & 11 & 15,3 & 12 & 10,0 \\
\hline $\begin{array}{l}\text { Zwangsgedanken mit religiösen oder solchen Inhalten, d } \\
\text { schlechtes Gewissen erzeugen* }\end{array}$ & 21 & 29,2 & 19 & 15,8 \\
\hline \multicolumn{4}{|l|}{ Zwangsgedanken, die sich auf Symmetrie oder Genauigkeit } & 18,3 \\
\hline Verschiedene Zwangsgedanken & 38 & 52,8 & 54 & 45,0 \\
\hline Zwangsgedanken in Bezug auf den eigenen Körper* & 27 & 37,5 & 29 & 24,2 \\
\hline Reinigungs-Wasch-Zwänge & 33 & 45,8 & 45 & 37,5 \\
\hline Kontrollzwänge & 38 & 52,8 & 55 & 45,8 \\
\hline Wiederholungszwänge & 30 & 41,7 & 38 & 31,7 \\
\hline Zählzwänge* & 19 & 26,4 & 16 & 13,3 \\
\hline Ordnungszwänge* & 18 & 25,0 & 14 & 11,7 \\
\hline Sammel- und Aufbewahrungszwänge & 12 & 16,7 & 13 & 10,8 \\
\hline Verschiedene Zwangshandlungen* & 39 & 54,2 & 38 & 31,7 \\
\hline
\end{tabular}

${ }^{*} \mathrm{p}<0,05$. rung könnten jedoch zu einer differentiellen Therapieindikation genutzt werden [Noam und Röper, 1999; Hemminger und Wewetzer, 2004].

Der Störungsbeginn in der Early-Onset-Gruppe lag bei durchschnittlich 11,3, in der Late-Onset-Gruppe bei durchschnittlich 26,5 Jahren. So zeigte sich bei unserer Stichprobe keine zweigipflige Verteilung wie bei Geller et al. [1998]. Über die Hälfte der Stichprobe gab einen Störungsbeginn zwischen dem 11. und 24. Lebensjahr an, ein deutlicher Peak des Störungsbeginns ist im 13. Lebensjahr erkennbar.

Logischerweise ergibt sich in kinder- und jugendpsychiatrischen Studien [Geller et al., 1998] ein jüngeres Durchschnittsalter bei Störungsbeginn, da jene Population, deren Störung erst im Erwachsenenalter beginnt, darin nicht enthalten ist. Bei der Geschlechterverteilung zwischen den Gruppen zeigte sich kein signifikant höherer Anteil an Männern in der EarlyOnset-Gruppe. Dieses Ergebnis steht im Einklang mit Befunden von Sobin et al. [2000] und Rosario-Campos et al. [2001], deren Studien ein ähnliches Design aufweisen. Allerdings steht das Ergebnis scheinbar in Widerspruch zu zahlreichen kinder- und jugendpsychiatrischen Studien, die ein Überwiegen des männlichen Anteils bei den Zwangsstörungen feststellten [Jans et al., 2003; Noshirvani et al., 1991; Thomsen, 1994]. Dieses Phänomen könnte durch eine höhere Komorbiditätsrate oder eine schwerere Krankheitssymptomatik bei den Jungen bedingt sein, was mit einer höheren Wahrscheinlichkeit zur klinischen Behandlung führt [Neudörfl et al., 1996]. Insgesamt lag der Schweregrad der Zwangsstörung (Y-BOCS-Werte) in der Gesamtstichprobe mit 23,95 Punkten an der Grenze zwischen mäßig und schwer ausgeprägter
Zwangsstörung. Die Early-Onset-Gruppe und die Late-OnsetGruppe unterscheiden sich diesbezüglich nicht. Jaisoorya et al. [2003] hingegen fanden sowohl bei der jugendlichen Stichprobe als auch bei der Stichprobe mit frühem Erkrankungsbeginn höhere Y-BOCS-Werte als bei der Erwachsenenstichprobe. Eine Analyse der Verteilung auf die einzelnen Schweregradbereiche ergab jedoch, dass bei den «extremen Zwangsstörungen», in deren Kategorie 12,8\% der Gesamtstichprobe fielen, mehr als doppelt so häufig Patienten mit frühem Störungsbeginn zu finden waren. Dies steht im Einklang mit klinischen Befunden, wonach Patienten, die nicht oder wenig vom Windacher Therapieansatz profitierten, (Y-BOCS-Reduktion $<30 \%$ ), ebenfalls gehäuft in der Early-Onset-Gruppe zu finden waren [Sedlmayr et al., 2004]. Betrachten wir die Skala zur Symptomatik der Zwangsstörung, so zeigt sich, dass die Early-Onset-Gruppe häufiger unter «Zwangsgedanken und -handlungen, gemischt» leidet als die Late-Onset-Gruppe. Die Gruppe mit frühem Störungsbeginn nannte in der Y-BOCS Symptom-Checkliste fast durchgängig mehr gegenwärtig vorhandene Leitsymptome als die Gruppe mit spätem Störungsbeginn. In der Gesamtstichprobe wurden durchschnittlich Symptome aus 7,41 von 15 möglichen Skalen genannt. Patienten mit frühem Störungsbeginn nannten durchschnittlich Symptome aus 8,15 Skalen, Patienten mit spätem Störungsbeginn Symptome aus 6,97 Skalen. Dieser Unterschied erwies sich als signifikant. Der Trend eines umfangreicheren Symptombildes zeigte sich auch in Bezug auf früher vorhandene Symptome, wobei für beide Gruppen die prozentualen Häufungen hier niedriger ausfielen.

Die Verteilung der ICD-10-Diagnosen und die unterschied- 
liche Zahl gegenwärtig und früher vorhandener Symptome weisen darauf hin, dass Personen mit frühem Störungsbeginn unter einer größeren Symptomvielfalt zu leiden scheinen als Personen mit spätem Störungsbeginn. Die vorliegende Untersuchung stellt eine explorative Studie dar, die Ergebnisse unterliegen daher einigen Einschränkungen. Zum einen bergen retrospektive Befragungen erwachsener Patienten die Gefahr, frühere Verhaltensprobleme zu pathologisieren. Der von uns festgelegte Cutoff-Wert von 15 Jahren stellt keine allgemein anerkannte Trennung zwischen frühem und spätem Störungsbeginn dar. Es wäre wünschenswert, einen Konsens über das Alter zu erreichen, das die Grenze des Early-Onset zum Late-Onset definiert, um Studienergebnisse miteinander vergleichen zu können.

Die Frage, ob es sich bei den Patienten mit frühem Störungsbeginn um eine abgrenzbare Subgruppe von Zwangspatienten handelt, konnte auch in dieser Untersuchung nicht geklärt werden. Ähnlichkeiten zwischen Patienten mit frühem und mit spätem Störungsbeginn, wie beispielsweise gleich hohe YBOCS-Werte, weisen auf einen Entwicklungstyp hin, der in die erwachsene Form der Zwangsstörung mündet [vgl. Thom- son, 1994; Geller et al., 1987, 2001]. Möglicherweise existiert jedoch in der Gruppe mit frühem Störungsbeginn eine Untergruppe, die eine größere Vielfalt an Symptomen und häufiger einen massiven Schweregrad der Störung aufweist. Diese Annahme kann eventuell mit zahlreichen neueren Studien, die sich dem Thema des höheren Einflusses neurobiologischer bzw. genetischer Substrate widmen, in Verbindung gebracht werden [Hegerl et al., 1999; Moll et al., 1999].

Die ermittelten Ergebnisse ermuntern in jedem Fall, den Forschungsaspekt des Early-Onset weiterzuverfolgen und die gefundenen phänotypischen Unterschiede mit Studien zum Therapieerfolg und individuellen Entstehungsgeschichten in Verbindung zu bringen. Langfristig könnte das Alter bei Störungsbeginn $\mathrm{zu}$ einem Kriterium für eine differentielle Therapieplanung hinsichtlich homogenerer Gruppen, des veranschlagten zeitlichen Rahmens oder auch des Medikamenteneinsatzes werden. In jedem Fall kommt dem Alter bei Störungsbeginn eine größere Bedeutung $\mathrm{zu}$ als vielleicht bisher angenommen wurde, und es sollte bereits in der Diagnostikphase stärker berücksichtigt werden.

\section{Literatur}

- Aigner M, Demal U, Zitterl W, Bach M, Trappl E, Lenz G: Verhaltenstherapeutische Gruppentherapie für Zwangsstörungen. Verhaltenstherapie 2004;14:7-14.

- Althaus D, Zaudig M, Hauke W, Röper G, Butollo W: Wirksamkeit eines spezifisch für Zwangsstörungen entwickelten stationären Gruppentherapiekonzepts bei gleichzeitiger Verhaltenstherapie und pharmakologischer Behandlung. Verhaltenstherapie 2000;10:16-23. Cohen J: Statistical Power Analysis for the Behavioural Sciences. Hillsdale, NY, Erlbaum, 1988.

Erikson EH: Identität und Lebenszyklus. Frankfurt/M., Suhrkamp, 1976.

Fontenelle L, Mendlowicz M, Marques C, Versiani M: Early- and late-onset obsessive-compulsive disorder in adult patients: an exploratory clinical and therapeutic study. J Psychiatr Res 2003;37:127-133.

- Geller D, Biedermann J, Jones J, Shapiro S, Schwartz S, Park KS: Obsessive-compulsive disorder in children and adolescents: a review. Harv Rev Psychiatry 1998;5: 260-273.

- Geller D, Biedermann J, Faraone S, Agranat A, Cradock C, Hagermoser L, Kim G, Frazier J, Coffey B: Developmental aspects of obsessive compulsive disorder: findings in children, adolescents, and adults. J Nerv Ment Dis 2001;189:471-477.

- Goodman WK, Rasmussen SA, Price LH, Mazure C, Henninger GR, Charnes DS: The Yale-Brown-Obsessive Compulsive Scale: Development, use, reliability. Arch Gen Psychiatry 1989;46:1006-1016.

Hand I, Büttner-Westphal H: Die Yale-Brown Obsessive Compulsive Scale (Y-BOCS). Ein halbstrukturiertes Interview zur Beurteilung des Schweregrades von Denk- und Handlungszwängen. Verhaltenstherapie 1991;3:223-233.

Hegerl U, Mavrogiorgou P: Die Zwangsstörung aus neurobiologischer Sicht. Verhaltensther Verhaltensmed 1999;20:435-447.

Hemminger U, Wewetzer C: Verhaltenstherapeutische Intervention bei Zwangsstörungen im Kindes- und Jugendalter; in Wewetzer C (Hrsg): Zwänge bei Kindern und Jugendlichen. Göttingen, Hogrefe, 2004, pp 97-107.
Holm S: A simple sequentially rejective multiple test procedure. Scand J Stat 1979;6:65-70.

Jaisoorya TS, Janardhan Reddy YC, Srinath S: Is juvenile obsessive-compulsive disorder a developmental subtype of the disorder? Findings from an Indian study. Eur Child Adolesc Psychiatry 2003;12:290-297. Jans T, Hemminger U, Wewetzer C: Der Verlauf von Zwangsstörungen mit Beginn im Kindes- und Jugendalter - eine Literaturübersicht. Z Kind Jugendpsychiatr Psychother 2003;31:187-201.

Karno M, Golding JM, Sorenson SB, Burnam MA: The epidemiology of obsessive compulsive disorder in five U.S. communities. Arch Gen Psychiatry 1988;45: 1094-1099.

Kegan R: Die Entwicklungsstufen des Selbst. München, Kindt, 1986.

Lakatos A, Reinecker H: Kognitive Verhaltenstherapie bei Zwangsstörungen. Ein Therapiemanual. Göttingen, Hogrefe, 1999.

Loevinger J: Ego Development: Conceptions and Theories. San Francisco, Jossey Bass, 1976.

Millet B, Kochman F, Gallarda T, Krebs MO, Demonfaucon F, Barrot I, Bourdel MC, Olié JP, Loo H, Hantouche EG: Phenomenological and comorbid features associated in obsessive-compulsive disorder: influence of age of onset. J Affect Disord 2004;798:241-246.

Moll GH, Hüther G, Rothenberger A: Neurobiologische Modellvorstellungen zu Entstehung und Aufhebung von Zwängen/Zwangsstörung. Verhaltensther Verhaltensmed 1999;20:449-463.

Neudörfl A, Herpertz-Dahlmann B: Der Verlauf der Zwangserkrankungen im Kindes- und Jugendalter eine Literaturübersicht. Z Kind Jugendpsychiatr 1996; 24:105-116.

Noam GG: Clinical Developmental Psychology: Toward developmentally differentiated interventions; in Damon W, Sigel I, Renninger KA (eds): Handbook of Child Psychology, ed 5. New York, Wiley, 1997, pp 585-614.
Noam GG, Röper G: Entwicklungsdiagnostik in klinisch-psychologischer Therapie und Forschung; in Oerter R, v. Hagen C, Röper G, Noam G (Hrsg): Klinische Entwicklungspsychologie. Weinheim, Beltz, 1999, pp 478-511.

Noshirvani H, Kasvikis Y, Marks I, Tsakiris F, Monteiro W: Gender-divergent aetiological factors in obsessive-compulsive disorder. Br J Psychiatry 1991;158: 260-263.

Rachmann S, Hodgson RJ: Obsessions and Compulsions. Englewood Cliffs, NY, Prentice-Hall, 1980

Rapoport J: Annotation Childhood Obsessive Compulsive Disorder. J Child Psychol Psychiatr 1986;27: 289-295.

Reinecker H: Zwänge - Diagnose, Theorien und Behandlung. Bern, Huber, 1994.

Reinecker H, Zaudig M: Langzeiteffekte bei der Behandlung von Zwangsstörungen. Windach Follow-up Studie. Lengerich, Pabst, 1995

Rosario-Campos MC, Leckman J, Mercadante M, Shavitt R, Silva Prado H, Sada P, Zamignani B, Miguel E: Adults with early-onset obsessive-compulsive disorder. Am J Psychiatry 2001;158:1899-1903.

Roth C, Siegl J, Aufdermauer N, Reinecker H: Therapie von Angst- und Zwangspatienten in der verhaltenstherapeutischen Praxis. Verhaltenstherapie 2004; 14:16-21.

Sedlmayr M, Röper G, Hauke, C, Piesbergen C, Zaudig M, Butollo W: Der frühe Beginn der Zwangsstörung - Einfluss auf die Therapie. 7. Jahrestagung der Deutschen Gesellschaft für Zwangserkrankungen, Lübeck, 2004.

Sobin C, Blundell ML, Karayiorgou M: Phenotypic differences in early- and late-onset obsessive-compulsive disorder. Compr Psychiatry 2000;41:373-379.

Thomsen PH: Obsessive-compulsive disorder in children and adolescents. a review of the literature. Eur Child Adolesc Psychiatry 1994;3:138-158. 